\title{
Construction of the General Solution of Planar Linear Discrete Systems with Constant Coefficients and Weak Delay
}

\author{
J. Diblík, ${ }^{1}$ D. Ya. Khusainov, ${ }^{2}$ and Z. Šmarda $^{1}$ \\ ${ }^{1}$ Brno University of Technology, Brno, Czech Republic \\ ${ }^{2}$ Kiev University, Kiev, Ukraine \\ Correspondence should be addressed to J. Diblík, diblik.j@fce.vutbr.cz
}

Received 19 January 2009; Accepted 30 March 2009

Recommended by Ulrich Krause

Planar linear discrete systems with constant coefficients and weak delay are considered. The characteristic equations of such systems are identical with those for the same systems but without delayed terms. In this case, the space of solutions with a given starting dimension is pasted after several steps into a space with dimension less than the starting one. In a sense this situation copies an analogous one known from the theory of linear differential systems with constant coefficients and weak delay when the initially infinite dimensional space of solutions on the initial interval on a reduced interval, turns (after several steps) into a finite dimensional set of solutions. For every possible case, general solutions are constructed and, finally, results on the dimensionality of the space of solutions are deduced.

Copyright (C 2009 J. Diblík et al. This is an open access article distributed under the Creative Commons Attribution License, which permits unrestricted use, distribution, and reproduction in any medium, provided the original work is properly cited.

\section{Introduction}

\subsection{Preliminary Notions and Properties}

We use the following notation: for integers $s, q, s \leq q$, we define $\mathbb{Z}_{s}^{q}:=\{s, s+1, \ldots, q\}$ where $s=-\infty$ or $q=\infty$ are admitted, too. Throughout this paper, using notation $\mathbb{Z}_{s}^{q}$, we always assume $s \leq q$. In this paper we deal with the discrete planar systems

$$
x(k+1)=A x(k)+B x(k-m),
$$

where $m \geq 0$ is a fixed integer, $k \in \mathbb{Z}_{0}^{\infty}, A=\left(a_{i j}\right)$ and $B=\left(b_{i j}\right)$ are constant $2 \times 2$ matrices, and $x: \mathbb{Z}_{-m}^{\infty} \rightarrow \mathbb{R}^{2}$. Following the terminology (used, e.g., in [1, 2]), (1.1) is referred to as a 
nondelayed discrete system if $m=0$ and as a delayed discrete system if $m>0$. Together with (1.1), we consider an initial (Cauchy) problem

$$
x(k)=\varphi(k),
$$

where $k=-m,-m+1, \ldots, 0$ with $\varphi: \mathbb{Z}_{-m}^{0} \rightarrow \mathbb{R}^{2}$. We will investigate only the case $m>0$ since the solution of (1.1) for $m=0$ is given by the known formula $x(k)=(A+B)^{k} \varphi(0)$ for $k \in \mathbb{Z}_{1}^{\infty}$.

The existence and uniqueness of the solution of the initial problems (1.1) and (1.2) on $\mathbb{Z}_{-m}^{\infty}$ are obvious. We recall that the solution $x: \mathbb{Z}_{-m}^{\infty} \rightarrow \mathbb{R}^{2}$ of (1.1) and (1.2) is defined as an infinite sequence

$$
\{x(-m)=\varphi(-m), x(-m+1)=\varphi(-m+1), \ldots, x(0)=\varphi(0), x(1), x(2), \ldots, x(k), \ldots\},
$$

such that, for any $k \in \mathbb{Z}_{0}^{\infty}$, equality (1.1) holds.

The space of all initial data (1.2) with $\varphi: \mathbb{Z}_{-m}^{0} \rightarrow \mathbb{R}^{2}$ is obviously $2(m+1)$-dimensional. Below we describe the fact that, among the systems (1.1), there are such systems that their space of solutions, being initially $2(m+1)$-dimensional, on a reduced interval turns into a space having dimension less than $2(m+1)$.

\subsection{Systems with Weak Delay}

We consider the system (1.1) and we look for a solution having the form $x(k)=\lambda^{k}, k \in \mathbb{Z}_{-m}^{\infty}$, $\lambda=$ const with a $\lambda \neq 0$. The usual procedure leads to a characteristic equation

$$
\operatorname{det}\left(A+\lambda^{-m} B-\lambda I\right)=0,
$$

where $I$ is the unit $2 \times 2$ matrix. Together with (1.1), we consider a system with the terms containing delays omitted

$$
x(k+1)=A x(k),
$$

and the characteristic equation

$$
\operatorname{det}(A-\lambda I)=0
$$

Definition 1.1. The system (1.1) is called a system with weak delay if the characteristic equations (1.4) and (1.6) corresponding to systems (1.1) and (1.5) are equal, that is, if for every $\lambda \in \mathbb{C} \backslash\{0\}$

$$
\operatorname{det}\left(A+\lambda^{-m} B-\lambda I\right)=\operatorname{det}(A-\lambda I)
$$


We consider a linear transformation

$$
x(k)=S y(k)
$$

with a nonsingular $2 \times 2$ matrix $\mathcal{S}$. Then the discrete system for $y$ is

$$
y(k+1)=A_{\mathcal{S}} y(k)+B_{\mathcal{S}} y(k-m)
$$

with $A_{\mathcal{S}}=\mathcal{S}^{-1} A \mathcal{S}, B_{\mathcal{S}}=\mathcal{S}^{-1} B \mathcal{S}$. We show that the property of a system to be the system with weak delay is preserved by every nonsingular linear transformation.

Lemma 1.2. If the system (1.1) is a system with weak delay, then its arbitrary linear nonsingular transformation (1.8) again leads to a system with the weak delay (1.9).

Proof. It is easy to show that

$$
\operatorname{det}\left(A_{\mathcal{S}}+\lambda^{-m} B_{\mathcal{S}}-\lambda I\right)=\operatorname{det}\left(A_{\mathcal{S}}-\lambda I\right)
$$

holds since

$$
\begin{aligned}
\operatorname{det}\left(A_{\mathcal{S}}+\lambda^{-m} B_{\mathcal{S}}-\lambda I\right) & =\operatorname{det}\left(\mathcal{S}^{-1} A \mathcal{S}+\lambda^{-m} \mathcal{S}^{-1} B \mathcal{S}-\lambda I\right) \\
& =\operatorname{det}\left[\mathcal{S}^{-1}\left(A+\lambda^{-m} B-\lambda I\right) \mathcal{S}\right] \\
& =\operatorname{det}\left(A+\lambda^{-m} B-\lambda I\right), \\
\operatorname{det}\left(A_{\mathcal{S}}-\lambda I\right) & =\operatorname{det}\left(\mathcal{S}^{-1} A \mathcal{S}-\lambda I\right) \\
& =\operatorname{det}\left[\mathcal{S}^{-1}(A-\lambda I) \mathcal{S}\right] \\
& =\operatorname{det}(A-\lambda I),
\end{aligned}
$$

and the equality

$$
\operatorname{det}\left(A+\lambda^{-m} B-\lambda I\right)=\operatorname{det}(A-\lambda I)
$$

is assumed.

\subsection{Necessary and Sufficient Conditions Determining the Weak Delay}

In the forthcoming theorem, we give conditions, in terms of determinants, indicating whether a system is a system with weak delay or not. 
Theorem 1.3. System (1.1) is a system with weak delay if and only if the following three conditions hold simultaneously:

$$
\begin{gathered}
b_{11}+b_{22}=0 \\
\left|\begin{array}{ll}
b_{11} & b_{12} \\
b_{21} & b_{22}
\end{array}\right|=0 \\
\left|\begin{array}{ll}
a_{11} & a_{12} \\
b_{21} & b_{22}
\end{array}\right|+\left|\begin{array}{ll}
b_{11} & b_{12} \\
a_{21} & a_{22}
\end{array}\right|=0 .
\end{gathered}
$$

Proof. We start with computing the determinant (1.4). We get

$$
\begin{aligned}
\operatorname{det}\left(A+\lambda^{-m} B-\lambda I\right)= & \left|\begin{array}{cc}
a_{11}+b_{11} \lambda^{-m}-\lambda & a_{12}+b_{12} \lambda^{-m} \\
a_{21}+b_{21} \lambda^{-m} & a_{22}+b_{22} \lambda^{-m}-\lambda
\end{array}\right| \\
= & \left|\begin{array}{cc}
a_{11}-\lambda & a_{12} \\
a_{21} & a_{22}-\lambda
\end{array}\right|-\lambda^{-m+1}\left(b_{11}+b_{22}\right) \\
& +\lambda^{-m}\left[\left|\begin{array}{ll}
a_{11} & a_{12} \\
b_{21} & b_{22}
\end{array}\right|+\left|\begin{array}{ll}
b_{11} & b_{12} \\
a_{21} & a_{22}
\end{array}\right|\right]+\lambda^{-2 m}\left|\begin{array}{ll}
b_{11} & b_{12} \\
b_{21} & b_{22}
\end{array}\right| .
\end{aligned}
$$

Now we see that, for (1.7) to hold, that is,

$$
\operatorname{det}\left(A+\lambda^{-m} B-\lambda I\right)=\operatorname{det}(A-\lambda I)=\left|\begin{array}{cc}
a_{11}-\lambda & a_{12} \\
a_{21} & a_{22}-\lambda
\end{array}\right|
$$

conditions (1.13) are both necessary and sufficient.

Remark 1.4. It is easy to see that conditions (1.13) are equivalent to

$$
\operatorname{tr} B=\operatorname{det} B=0, \quad \operatorname{det}(A+B)=\operatorname{det} A .
$$

\subsection{Problem under Consideration}

The aim of this paper is to show that the dimension of the space of all solutions, being initially equal to the dimension $2(m+1)$ of the space of initial data (1.2) generated by discrete functions $\varphi$, is, after several steps, reduced (on an interval of the form $\mathbb{Z}_{s}^{\infty}$ with an $s>0$ ) to a dimension less than the initial one. In other words, we will show that the $2(m+1)$-dimensional space of all solutions of (1.1) is reduced to a less-dimensional space of solutions on $\mathbb{Z}_{s}^{\infty}$. This problem is solved directly by explicitly computing the corresponding solutions of the Cauchy problems with each of the cases arising being considered. The underlying idea for such investigation is simple. If (1.1) is a system with weak delay, then the corresponding characteristic equation has only two eigenvalues instead of $2(m+1)$ eigenvalues in the case of systems with nonweak 
delay. This explains why the dimension of the space of solutions becomes less than the initial one. The final results (Theorems 2.5-2.8) provide the dimension of the space of solutions.

\subsection{Auxiliary Formula}

For the reader's convenience we recall one explicit formula (see, e.g., [3]) for the solutions of linear scalar discrete nondelayed equations used in this paper. We consider the first-order linear discrete nonhomogeneous equation

$$
w(k+1)=a w(k)+g(k), \quad w\left(k_{0}\right)=w_{0}, \quad k \in \mathbb{Z}_{k_{0}}^{\infty}
$$

with $a \in \mathbb{C}$ and $g: \mathbb{Z}_{k_{0}}^{\infty} \rightarrow \mathbb{C}$. Then it is easy to verify that

$$
w(k)=a^{k-k_{0}} w_{0}+\sum_{r=k_{0}}^{k-1} a^{k-1-r} g(r), \quad k \in \mathbb{Z}_{k_{0}+1}^{\infty}
$$

Throughout the paper, we adopt the customary notation for the sum: $\sum_{i=\ell+s}^{\ell} \mathcal{F}(i)=0$ where $\ell$ is an integer, $s$ is a positive integer and, " $F$ " denotes the function considered independently of whether it is defined for indicated arguments or not.

\section{Results}

If (1.7) holds, then (1.4) and (1.6) have only two (and the same) roots simultaneously. In order to prove the properties of the family of solutions of (1.1) formulated in Section 1.4, we will separately discuss all the possible combinations of roots, that is, the cases of two real and distinct roots, a couple of complex conjugate roots, and, finally, a two-fold real root.

\subsection{Jordan Forms of Matrix A and Corresponding Solutions of the Problem (1.1), (1.2)}

It is known that, for every matrix $A$, there exists a nonsingular matrix $S$ transforming it to the corresponding Jordan matrix form $\Lambda$. This means that

$$
\Lambda=S^{-1} A S,
$$

where $\Lambda$ has the following possible forms, depending on the roots of the characteristic equation (1.6), that is, on the roots of

$$
\lambda^{2}-\left(a_{11}+a_{22}\right) \lambda+\left(a_{11} a_{22}-a_{12} a_{21}\right)=0 .
$$


If (2.2) has two real distinct roots $\lambda_{1}, \lambda_{2}$, then

$$
\Lambda=\left(\begin{array}{ll}
\lambda_{1} & 0 \\
0 & \lambda_{2}
\end{array}\right)
$$

if the roots are complex conjugate, that is, $\lambda_{1,2}=p \pm i q$ with $q \neq 0$, then

$$
\Lambda=\left(\begin{array}{cc}
p & q \\
-q & p
\end{array}\right)
$$

and, finally, in the case of one two-fold real root $\lambda_{1,2}=\lambda$, we have either

$$
\Lambda=\left(\begin{array}{ll}
\lambda & 0 \\
0 & \lambda
\end{array}\right)
$$

or

$$
\Lambda=\left(\begin{array}{ll}
\lambda & 1 \\
0 & \lambda
\end{array}\right)
$$

The transformation $y(k)=S^{-1} x(k)$ transforms (1.1) into a system

$$
y(k+1)=\Lambda y(k)+B^{*} y(k-m), \quad k \in \mathbb{Z}_{0}^{\infty},
$$

with $B^{*}=S^{-1} B S, B^{*}=\left(b_{i j}^{*}\right), i, j=1,2$. Together with (2.7), we consider an initial problem

$$
y(k)=\varphi^{*}(k),
$$

$k \in \mathbb{Z}_{-m}^{0}$ with $\varphi^{*}: \mathbb{Z}_{-m}^{0} \rightarrow \mathbb{R}^{2}$ where $\varphi^{*}(k)=S^{-1} \varphi(k)$ is the initial function corresponding to the initial function $\varphi$ in (1.2).

Below we consider all four possible cases (2.3)-(2.6) separately.

We define

$$
\Phi_{1}(k):=\left(0, \varphi_{1}^{*}(k)\right)^{T}, \quad \Phi_{2}(k):=\left(\varphi_{2}^{*}(k), 0\right)^{T}, \quad k \in \mathbb{Z}_{-m}^{0} .
$$

Assuming that the system (1.1) is a system with weak delay, the system (2.7), due to Lemma 1.2 , is a system with weak delay again. 


\subsubsection{The Case (2.3) of Two Real Distinct Roots}

In this case, we have $\Lambda^{k}=\operatorname{diag}\left(\lambda_{1}^{k}, \lambda_{2}^{k}\right)$. The necessary and sufficient conditions (1.13) for (2.7) turn into

$$
\begin{gathered}
b_{11}^{*}+b_{22}^{*}=0 \\
\left|\begin{array}{ll}
b_{11}^{*} & b_{12}^{*} \\
b_{21}^{*} & b_{22}^{*}
\end{array}\right|=b_{11}^{*} b_{22}^{*}-b_{12}^{*} b_{21}^{*}=0 \\
\left|\begin{array}{cc}
\lambda_{1} & 0 \\
b_{21}^{*} & b_{22}^{*}
\end{array}\right|+\left|\begin{array}{cc}
b_{11}^{*} & b_{12}^{*} \\
0 & \lambda_{2}
\end{array}\right|=\lambda_{1} b_{22}^{*}+\lambda_{2} b_{11}^{*}=0
\end{gathered}
$$

Since $\lambda_{1} \neq \lambda_{2},(2.10)$, (2.12) yield $b_{11}^{*}=b_{22}^{*}=0$. Then, from (2.11), we get $b_{12}^{*} b_{21}^{*}=0$, so either $b_{21}^{*}=0$ or $b_{12}^{*}=0$.

Theorem 2.1. Let (1.1) be a system with weak delay and (2.2) admit two real distinct roots $\lambda_{1}, \lambda_{2}$. Then $b_{11}^{*}=b_{22}^{*}=b_{12}^{*} b_{21}^{*}=0$. The solution of the initial problems (1.1) and (1.2) is $x(k)=S y(k)$, $k \in \mathbb{Z}_{-m}^{\infty}$ where $y(k)$ has, in the case $b_{21}^{*}=0$, the form

$$
y(k)= \begin{cases}\varphi^{*}(k), & \text { if } k \in \mathbb{Z}_{-m}^{0}, \\ \Lambda^{k} \varphi^{*}(0)+b_{12}^{*} \sum_{r=0}^{k-1} \lambda_{1}^{k-1-r} \Phi_{2}(r-m), & \text { if } k \in \mathbb{Z}_{1}^{m+1}, \\ \Lambda^{k} \varphi^{*}(0)+b_{12}^{*}\left[\sum_{r=0}^{m} \lambda_{1}^{k-1-r} \Phi_{2}(r-m)+\Phi_{2}(0) \sum_{r=m+1}^{k-1} \lambda_{1}^{k-1-r} \lambda_{2}^{r-m}\right], & \text { if } k \in \mathbb{Z}_{m+2}^{\infty}\end{cases}
$$

and, in the case $b_{12}^{*}=0$, the form

$$
y(k)= \begin{cases}\varphi^{*}(k), & \text { if } k \in \mathbb{Z}_{-m}^{0}, \\ \Lambda^{k} \varphi^{*}(0)+b_{21}^{*} \sum_{r=0}^{k-1} \lambda_{2}^{k-1-r} \Phi_{1}(r-m), & \text { if } k \in \mathbb{Z}_{1}^{m+1}, \\ \Lambda^{k} \varphi^{*}(0)+b_{21}^{*}\left[\sum_{r=0}^{m} \lambda_{2}^{k-1-r} \Phi_{1}(r-m)+\Phi_{1}(0) \sum_{r=m+1}^{k-1} \lambda_{1}^{r-m} \lambda_{2}^{k-1-r}\right], & \text { if } k \in \mathbb{Z}_{m+2}^{\infty} .\end{cases}
$$

Proof. In the case considered we have $b_{11}^{*}=b_{22}^{*}=b_{12}^{*} b_{21}^{*}=0$ and the transformed system (2.7) takes either the form

$$
\begin{aligned}
& y_{1}(k+1)=\lambda_{1} y_{1}(k)+b_{12}^{*} y_{2}(k-m), \quad k \in \mathbb{Z}_{0}^{\infty}, \\
& y_{2}(k+1)=\lambda_{2} y_{2}(k), \quad k \in \mathbb{Z}_{0}^{\infty},
\end{aligned}
$$


if $b_{21}^{*}=0$ or the form

$$
\begin{aligned}
& y_{1}(k+1)=\lambda_{1} y_{1}(k), \quad k \in \mathbb{Z}_{0}^{\infty}, \\
& y_{2}(k+1)=\lambda_{2} y_{2}(k)+b_{21}^{*} y_{1}(k-m), \quad k \in \mathbb{Z}_{0}^{\infty},
\end{aligned}
$$

if $b_{12}^{*}=0$. We investigate only the initial problem (2.15), (2.16), (2.8) since the initial problem (2.17), (2.18), (2.8) can be examined in a similar way. From (2.16) and (2.8), we get

$$
y_{2}(k)= \begin{cases}\varphi_{2}^{*}(k), & \text { if } k \in \mathbb{Z}_{-m}^{0}, \\ \lambda_{2}^{k} \varphi_{2}^{*}(0), & \text { if } k \in \mathbb{Z}_{1}^{\infty}\end{cases}
$$

Then (2.15) becomes

$$
y_{1}(k+1)= \begin{cases}\lambda_{1} y_{1}(k)+b_{12}^{*} \varphi_{2}^{*}(k-m), & \text { if } k \in \mathbb{Z}_{0}^{m}, \\ \lambda_{1} y_{1}(k)+b_{12}^{*} \lambda_{2}^{k-m} \varphi_{2}^{*}(0), & \text { if } k \in \mathbb{Z}_{m+1}^{\infty}\end{cases}
$$

First we solve this equation for $k \in \mathbb{Z}_{0}^{m}$. This means that we consider the problem

$$
\begin{gathered}
y_{1}(k+1)=\lambda_{1} y_{1}(k)+b_{12}^{*} \varphi_{2}^{*}(k-m), \quad k \in \mathbb{Z}_{0}^{\infty}, \\
y_{1}(0)=\varphi_{1}^{*}(0) .
\end{gathered}
$$

With the aid of formula (1.18), we get

$$
y_{1}(k)=\lambda_{1}^{k} \varphi_{1}^{*}(0)+b_{12}^{*} \sum_{r=0}^{k-1} \lambda_{1}^{k-1-r} \varphi_{2}^{*}(r-m), \quad k \in \mathbb{Z}_{1}^{m+1}
$$

Now we solve (2.20) for $k \in \mathbb{Z}_{m+1}^{\infty}$, that is, we consider the problem (with initial data deduced from (2.22))

$$
\begin{gathered}
y_{1}(k+1)=\lambda_{1} y_{1}(k)+b_{12}^{*} \lambda_{2}^{k-m} \varphi_{2}^{*}(0), \quad k \in \mathbb{Z}_{m+1}^{\infty}, \\
y_{1}(m+1)=\lambda_{1}^{m+1} \varphi_{1}^{*}(0)+b_{12}^{*} \sum_{r=0}^{m} \lambda_{1}^{m-r} \varphi_{2}^{*}(r-m) .
\end{gathered}
$$


Applying formula (1.18) yields (for $k \in \mathbb{Z}_{m+2}^{\infty}$ )

$$
\begin{aligned}
y_{1}(k) & =\lambda_{1}^{k-(m+1)} y_{1}(m+1)+b_{12}^{*} \varphi_{2}^{*}(0) \sum_{r=m+1}^{k-1} \lambda_{1}^{k-1-r} \lambda_{2}^{r-m} \\
& =\lambda_{1}^{k-(m+1)}\left[\lambda_{1}^{m+1} \varphi_{1}^{*}(0)+b_{12}^{*} \sum_{r=0}^{m} \lambda_{1}^{m-r} \varphi_{2}^{*}(r-m)\right]+b_{12}^{*} \varphi_{2}^{*}(0) \sum_{r=m+1}^{k-1} \lambda_{1}^{k-1-r} \lambda_{2}^{r-m} \\
& =\lambda_{1}^{k} \varphi_{1}^{*}(0)+b_{12}^{*} \sum_{r=0}^{m} \lambda_{1}^{k-1-r} \varphi_{2}^{*}(r-m)+b_{12}^{*} \varphi_{2}^{*}(0) \sum_{r=m+1}^{k-1} \lambda_{1}^{k-1-r} \lambda_{2}^{r-m} .
\end{aligned}
$$

Picking up all particular cases (2.8), (2.22), and (2.24), we have

$$
y_{1}(k)= \begin{cases}\varphi_{1}^{*}(k), & \text { if } k \in \mathbb{Z}_{-m}^{0}, \\ \lambda_{1}^{k} \varphi_{1}^{*}(0)+b_{12}^{*} \sum_{r=0}^{k-1} \lambda_{1}^{k-1-r} \varphi_{2}^{*}(r-m), & \text { if } k \in \mathbb{Z}_{1}^{m+1}, \\ \lambda_{1}^{k} \varphi_{1}^{*}(0)+b_{12}^{*}\left[\sum_{r=0}^{m} \lambda_{1}^{k-1-r} \varphi_{2}^{*}(r-m)+\varphi_{2}^{*}(0) \sum_{r=m+1}^{k-1} \lambda_{1}^{k-1-r} \lambda_{2}^{r-m}\right], & \text { if } k \in \mathbb{Z}_{m+2}^{\infty} .\end{cases}
$$

Now, taking into account (2.9), the formula (2.13) is a consequence of (2.19) and (2.25). The formula (2.14) can be proved in a similar way.

Finally, we note that both formulas (2.13) and (2.14) remain valid for $b_{12}^{*}=b_{21}^{*}=0$ as well. In this case, the transformed system (2.7) reduces to a system without delay.

\subsubsection{The Case (2.4) of Two Complex Conjugate Roots}

The necessary and sufficient conditions (1.13) for (2.7) take the forms (2.10) and (2.11) and

$$
\left|\begin{array}{cc}
p & q \\
b_{21}^{*} & b_{22}^{*}
\end{array}\right|+\left|\begin{array}{cc}
b_{11}^{*} & b_{12}^{*} \\
-q & p
\end{array}\right|=p\left(b_{11}^{*}+b_{22}^{*}\right)+q\left(b_{12}^{*}-b_{21}^{*}\right)=0 .
$$

The system of conditions (2.10), (2.11), and (2.26) gives $b_{12}^{*}=b_{21}^{*}\left(b_{11}^{*}\right)^{2}=-\left(b_{12}^{*}\right)^{2}$ and admits only one possibility, namely,

$$
b_{11}^{*}=b_{22}^{*}=b_{12}^{*}=b_{21}^{*}=0 \text {. }
$$

Consequently, $B^{*}=0$ and $B=0$ as well. The initial problems (1.1) and (1.2) reduces to a problem without delay

$$
\begin{gathered}
x(k+1)=A x(k), \quad k \in \mathbb{Z}_{-m}^{0}, \\
x(k)=\varphi(k), \quad k \in \mathbb{Z}_{-m}^{0},
\end{gathered}
$$


and, obviously,

$$
x(k)= \begin{cases}\varphi(k), & \text { if } k \in \mathbb{Z}_{-m}^{0}, \\ A^{k} \varphi(0), & \text { if } k \in \mathbb{Z}_{1}^{\infty} .\end{cases}
$$

\subsubsection{The Case (2.5) of Two-Fold Real Root}

We have $\Lambda^{k}=\operatorname{diag}\left(\lambda^{k}, \lambda^{k}\right)$. The necessary and sufficient conditions (1.13) are, for (2.7), reduced to (2.10), (2.11), and

$$
\left|\begin{array}{cc}
\lambda & 0 \\
b_{21}^{*} & b_{22}^{*}
\end{array}\right|+\left|\begin{array}{cc}
b_{11}^{*} & b_{12}^{*} \\
0 & \lambda
\end{array}\right|=\lambda\left(b_{11}^{*}+b_{22}^{*}\right)=0 .
$$

From (2.10), (2.11), and (2.30), we get $b_{12}^{*} b_{21}^{*}=-\left(b_{11}^{*}\right)^{2}$. Now we will analyse the two possible cases: $b_{12}^{*} b_{21}^{*}=0$ and $b_{12}^{*} b_{21}^{*} \neq 0$.

\section{The Case $b_{12}^{*} b_{21}^{*}=0$}

Theorem 2.2. Let (1.1) be a system with weak delay, (2.2) admit a two-fold root $\lambda_{1,2}=\lambda, b_{12}^{*} b_{21}^{*}=0$ and the matrix $\Lambda$ has the form (2.5). Then the solution of the initial problems (1.1) and (1.2) is $x(k)=S y(k), k \in \mathbb{Z}_{-m}^{\infty}$ where $y(k)$ has, in the case $b_{21}^{*}=0$, the form

$$
y(k)= \begin{cases}\varphi^{*}(k), & \text { if } k \in \mathbb{Z}_{-m}^{0}, \\ \Lambda^{k} \varphi^{*}(0)+b_{12}^{*} \sum_{r=0}^{k-1} \lambda^{k-1-r} \Phi_{2}(r-m), & \text { if } k \in \mathbb{Z}_{1}^{m+1}, \\ \Lambda^{k} \varphi^{*}(0)+b_{12}^{*}\left[\sum_{r=0}^{m} \lambda^{k-1-r} \Phi_{2}(r-m)+(k-1-m) \lambda^{k-1-m} \Phi_{2}(0)\right], & \text { if } k \in \mathbb{Z}_{m+2}^{\infty}\end{cases}
$$

and, in the case $b_{12}^{*}=0$, the form

$$
y(k)= \begin{cases}\varphi^{*}(k), & \text { if } k \in \mathbb{Z}_{-m}^{0}, \\ \Lambda^{k} \varphi^{*}(0)+b_{21}^{*} \sum_{r=0}^{k-1} \lambda^{k-1-r} \Phi_{1}(r-m), & \text { if } k \in \mathbb{Z}_{1}^{m+1}, \\ \Lambda^{k} \varphi^{*}(0)+b_{21}^{*}\left[\sum_{r=0}^{m} \lambda^{k-1-r} \Phi_{1}(r-m)+(k-1-m) \lambda^{k-1-m} \Phi_{1}(0)\right], & \text { if } k \in \mathbb{Z}_{m+2}^{\infty} .\end{cases}
$$

Proof. The assumption $b_{21}^{*}=0$ or $b_{12}^{*}=0$ leads to $b_{11}^{*}=b_{22}^{*}=0$. Then the following cases arise. Either $b_{12}^{*} \neq 0, b_{21}^{*}=0$ or $b_{12}^{*}=0, b_{21}^{*} \neq 0$ or $b_{12}^{*}=b_{21}^{*}=0$. The latter case is covered by the above 
formulas (2.31) and (2.32) since it can be treated as system (2.28) considered previously (with $A=\Lambda$ ) when $B^{*}=B=0$, and the corresponding solution is described by the formula (2.29). If $b_{12}^{*} \neq 0$, then (2.7) turns into the system

$$
\begin{aligned}
& y_{1}(k+1)=\lambda y_{1}(k)+b_{12}^{*} y_{2}(k-m), \quad k \in \mathbb{Z}_{0}^{\infty}, \\
& y_{2}(k+1)=\lambda y_{2}(k), \quad k \in \mathbb{Z}_{0}^{\infty}
\end{aligned}
$$

and, if $b_{21}^{*} \neq 0$, then (2.7) turns into the system

$$
\begin{aligned}
& y_{1}(k+1)=\lambda y_{1}(k), \quad k \in \mathbb{Z}_{0}^{\infty}, \\
& y_{2}(k+1)=\lambda y_{2}(k)+b_{21}^{*} y_{1}(k-m), \quad k \in \mathbb{Z}_{0}^{\infty} .
\end{aligned}
$$

System (2.33) can be solved in much the same way as the systems (2.15) and (2.16) if we put $\lambda_{1}=\lambda_{2}=\lambda$, and the discussion of the system (2.34) copies the discussion of the systems (2.17) and (2.18) with $\lambda_{1}=\lambda_{2}=\lambda$. Formulas (2.31) and (2.32) are consequences of (2.13) and (2.14).

The Case $b_{12}^{*} b_{21}^{*} \neq 0$

For $k \in \mathbb{Z}_{-m}^{0}$ we define

$$
\Phi(k):=\left(b_{11}^{*}\left[\varphi_{1}^{*}(k)+\frac{b_{12}^{*}}{b_{11}^{*}} \varphi_{2}^{*}(k)\right],-\frac{\left(b_{11}^{*}\right)^{2}}{b_{12}^{*}}\left[\varphi_{1}^{*}(k)+\frac{b_{12}^{*}}{b_{11}^{*}} \varphi_{2}^{*}(k)\right]\right)^{T} .
$$

Theorem 2.3. Let the system (1.1) be a system with weak delay, (2.2) admit two repeated roots $\lambda_{1,2}=\lambda, b_{12}^{*} b_{21}^{*} \neq 0$, and the matrix $\Lambda$ has the form (2.5). Then the solution of the initial problems (1.1) and (1.2) is given by $x(k)=S y(k), k \in \mathbb{Z}_{-m}^{\infty}$ where $y(k)$ has the form

$$
y(k)= \begin{cases}\varphi^{*}(k), & \text { if } k \in \mathbb{Z}_{-m}^{0}, \\ \Lambda^{k} \varphi^{*}(0)+\sum_{r=0}^{k-1} \lambda^{k-1-r} \Phi(r-m), & \text { if } k \in \mathbb{Z}_{1}^{m+1}, \\ \Lambda^{k} \varphi^{*}(0)+\sum_{r=0}^{m} \lambda^{k-1-r} \Phi(r-m)+(k-1-m) \lambda^{k-1-m} \Phi(0), & \text { if } k \in \mathbb{Z}_{m+2}^{\infty} .\end{cases}
$$

Proof. In this case, all the entries of $B^{*}$ are nonzero and, from (2.10), (2.11), and (2.30), we get

$$
B^{*}=\left(\begin{array}{cc}
b_{11}^{*} & b_{12}^{*} \\
-\frac{\left(b_{11}^{*}\right)^{2}}{b_{12}^{*}} & -b_{11}^{*}
\end{array}\right) \text {. }
$$


Then the system (2.7) reduces to

$$
\begin{gathered}
y_{1}(k+1)=\lambda y_{1}(k)+b_{11}^{*} y_{1}(k-m)+b_{12}^{*} y_{2}(k-m), \\
y_{2}(k+1)=\lambda y_{2}(k)-\frac{\left(b_{11}^{*}\right)^{2}}{b_{12}^{*}} y_{1}(k-m)-b_{11}^{*} y_{2}(k-m),
\end{gathered}
$$

where $k \in \mathbb{Z}_{0}^{\infty}$. It is easy to see (multiplying (2.39) by $b_{12}^{*} / b_{11}^{*}$ and summing both equations) that

$$
y_{1}(k+1)+\frac{b_{12}^{*}}{b_{11}^{*}} y_{2}(k+1)=\lambda\left[y_{1}(k)+\frac{b_{12}^{*}}{b_{11}^{*}} y_{2}(k)\right], \quad k \in \mathbb{Z}_{0}^{\infty}
$$

We can see (2.40) as a homogeneous equation with respect to the unknown expression $y_{1}(k)+$ $\left(b_{12}^{*} / b_{11}^{*}\right) y_{2}(k)$. Then, using (1.18), we obtain

$$
y_{1}(k)+\frac{b_{12}^{*}}{b_{11}^{*}} y_{2}(k)= \begin{cases}\varphi_{1}^{*}(k)+\frac{b_{12}^{*}}{b_{11}^{*}} \varphi_{2}^{*}(k), & \text { if } k \in \mathbb{Z}_{-m}^{0}, \\ \lambda^{k}\left[\varphi_{1}^{*}(0)+\frac{b_{12}^{*}}{b_{11}^{*}} \varphi_{2}^{*}(0)\right], & \text { if } k \in \mathbb{Z}_{1}^{\infty} .\end{cases}
$$

With the aid of (2.41), we rewrite the systems (2.38) and (2.39) as follows:

$$
\begin{aligned}
& y_{1}(k+1)= \begin{cases}\lambda y_{1}(k)+b_{11}^{*}\left[\varphi_{1}^{*}(k-m)+\frac{b_{12}^{*}}{b_{11}^{*}} \varphi_{2}^{*}(k-m)\right], & \text { if } k \in \mathbb{Z}_{0}^{m}, \\
\lambda y_{1}(k)+b_{11}^{*} \lambda^{k-m}\left[\varphi_{1}^{*}(0)+\frac{b_{12}^{*}}{b_{11}^{*}} \varphi_{2}^{*}(0)\right], & \text { if } k \in \mathbb{Z}_{m+1}^{\infty},\end{cases} \\
& y_{2}(k+1)= \begin{cases}\lambda y_{2}(k)-\frac{\left(b_{11}^{*}\right)^{2}}{b_{12}^{*}}\left[\varphi_{1}^{*}(k-m)+\frac{b_{12}^{*}}{b_{11}^{*}} \varphi_{2}^{*}(k-m)\right], & \text { if } k \in \mathbb{Z}_{0}^{m}, \\
\lambda y_{2}(k)-\frac{\left(b_{11}^{*}\right)^{2}}{b_{12}^{*}} \lambda^{k-m}\left[\varphi_{1}^{*}(0)+\frac{b_{12}^{*}}{b_{11}^{*}} \varphi_{2}^{*}(0)\right], & \text { if } k \in \mathbb{Z}_{m+1}^{\infty} .\end{cases}
\end{aligned}
$$


It is easy to see that the system (2.42) is decomposed into two separate equations. Solving each of them in a similar way as in the proof of Theorem 2.1 using (1.18) (details are omitted), we conclude

$$
\begin{aligned}
& y_{1}(k)=\left\{\begin{aligned}
\varphi_{1}^{*}(k), & \text { if } k \in \mathbb{Z}_{-m}^{0}, \\
\lambda^{k} \varphi_{1}^{*}(0)+b_{11}^{*} \sum_{r=0}^{k-1} \lambda^{k-1-r}\left[\varphi_{1}^{*}(r-m)+\frac{b_{12}^{*}}{b_{11}^{*}} \varphi_{2}^{*}(r-m)\right], & \text { if } k \in \mathbb{Z}_{1}^{m+1}, \\
\lambda^{k} \varphi_{1}^{*}(0)+b_{11}^{*}\left[\sum_{r=0}^{m} \lambda^{k-1-r}\left[\varphi_{1}^{*}(r-m)+\frac{b_{12}^{*}}{b_{11}^{*}} \varphi_{2}^{*}(r-m)\right]\right. & \\
\left.+(k-1-m) \lambda^{k-1-m}\left[\varphi_{1}^{*}(0)+\frac{b_{12}^{*}}{b_{11}^{*}} \varphi_{2}^{*}(0)\right]\right], & \text { if } k \in \mathbb{Z}_{m+2}^{\infty}
\end{aligned}\right. \\
& y_{2}(k)=\left\{\begin{array}{cc}
\varphi_{2}^{*}(k) & \text { if } k \in \mathbb{Z}_{-m}^{0}, \\
\lambda^{k} \varphi_{2}^{*}(0)-\frac{\left(b_{11}^{*}\right)^{2}}{b_{12}^{*}} \sum_{r=0}^{k-1} \lambda^{k-1-r}\left[\varphi_{1}^{*}(r-m)+\frac{b_{12}^{*}}{b_{11}^{*}} \varphi_{2}^{*}(r-m)\right], & \text { if } k \in \mathbb{Z}_{1}^{m+1}, \\
\lambda^{k} \varphi_{2}^{*}(0)-\frac{\left(b_{11}^{*}\right)^{2}}{b_{12}^{*}}\left[\sum_{r=0}^{m} \lambda^{k-1-r}\left[\varphi_{1}^{*}(r-m)+\frac{b_{12}^{*}}{b_{11}^{*}} \varphi_{2}^{*}(r-m)\right]\right. & \\
\left.+(k-1-m) \lambda^{k-1-m}\left[\varphi_{1}^{*}(0)+\frac{b_{12}^{*}}{b_{11}^{*}} \varphi_{2}^{*}(0)\right]\right], & \text { if } k \in \mathbb{Z}_{m+2}^{\infty} .
\end{array}\right.
\end{aligned}
$$

Formula (2.36) is now a direct consequence of (2.43) and (2.35).

\subsubsection{The Case (2.6) of Two-Fold Real Root}

If the matrix $\Lambda$ has the form (2.6), the necessary and sufficient conditions (1.13), for (2.7), are reduced to (2.10), (2.11), and

$$
\left|\begin{array}{cc}
\lambda & 1 \\
b_{21}^{*} & b_{22}^{*}
\end{array}\right|+\left|\begin{array}{cc}
b_{11}^{*} & b_{12}^{*} \\
0 & \lambda
\end{array}\right|=\lambda\left(b_{11}^{*}+b_{22}^{*}\right)-b_{21}^{*}=0 .
$$

Then (2.10), (2.11), and (2.44) give $b_{11}^{*}=b_{22}^{*}=b_{21}^{*}=0$, and the system (2.7) can be written as

$$
\begin{aligned}
& y_{1}(k+1)=\lambda y_{1}(k)+y_{2}(k)+b_{12}^{*} y_{2}(k-m), \quad k \in \mathbb{Z}_{0}^{\infty}, \\
& y_{2}(k+1)=\lambda y_{2}(k), \quad k \in \mathbb{Z}_{0}^{\infty} .
\end{aligned}
$$

Solving (2.46), we get

$$
y_{2}(k)= \begin{cases}\varphi_{2}^{*}(k), & \text { if } k \in \mathbb{Z}_{-m}^{0}, \\ \lambda^{k} \varphi_{2}^{*}(0), & \text { if } k \in \mathbb{Z}_{1}^{\infty}\end{cases}
$$


Then (2.45) turns into

$$
y_{1}(k+1)= \begin{cases}\lambda y_{1}(k)+\lambda^{k} \varphi_{2}^{*}(0)+b_{12}^{*} \varphi_{2}^{*}(k-m), & \text { if } k \in \mathbb{Z}_{0}^{m} \\ \lambda y_{1}(k)+\lambda^{k} \varphi_{2}^{*}(0)+b_{12}^{*} \lambda^{k-m} \varphi_{2}^{*}(0), & \text { if } k \in \mathbb{Z}_{m+1}^{\infty}\end{cases}
$$

Equation (2.48) can be solved in a similar way as in the proof of Theorem 2.1 using (1.18) (we omit details). We get

$$
y_{1}(k)= \begin{cases}\varphi_{1}^{*}(k), & \text { if } k \in \mathbb{Z}_{-m}^{0}, \\ \lambda^{k} \varphi_{1}^{*}(0)+\sum_{r=0}^{k-1} \lambda^{k-1-r}\left[\lambda^{r} \varphi_{2}^{*}(0)+b_{12}^{*} \varphi_{2}^{*}(r-m)\right], & \text { if } k \in \mathbb{Z}_{1}^{m+1}, \\ \lambda^{k} \varphi_{1}^{*}(0)+k \lambda^{k-1} \varphi_{2}^{*}(0)+b_{12}^{*}(k-1-m) \lambda^{k-1-m} \varphi_{2}^{*}(0) & \\ +b_{12}^{*} \sum_{r=0}^{m} \lambda^{k-1-r} \varphi_{2}^{*}(r-m), & \text { if } k \in \mathbb{Z}_{m+2}^{\infty} .\end{cases}
$$

Formulas (2.47), (2.49) can be used in the case $b_{12}^{*}=0$ as well. In this way, the ensuing result is proved.

Theorem 2.4. Let (1.1) be a system with weak delay, (2.2) admit two repeated roots $\lambda_{1,2}=\lambda$, and the matrix $\Lambda$ has the form (2.6). Then $b_{11}^{*}=b_{22}^{*}=b_{21}^{*}=0$ and the solution of the initial problems (1.1) and (1.2) is $x(k)=S y(k), k \in \mathbb{Z}_{-m}^{\infty}$ where $y(k)=\left(y_{1}(k), y_{2}(k)\right)^{T}, y_{1}(k)$ is defined by (2.49) and $y_{2}(k)$ by $(2.47)$.

\subsection{Dimension of the Set of Solutions}

Since all the possible cases of the planar system (1.1) with weak delay have been analysed, we are ready to formulate results concerning the dimension of the space of solutions of (1.1) assuming that initial conditions (1.2) are variable.

Theorem 2.5. Let (1.1) be a system with weak delay, and (2.2) has both roots different from zero. Then the space of solutions, being initially $2(m+1)$-dimensional, becomes on $\mathbb{Z}_{m+2}^{\infty}$ only

(1) $(m+2)$-dimensional if (2.2) has
(a) two real distinct roots and $\left(b_{12}^{*}\right)^{2}+\left(b_{21}^{*}\right)^{2}>0$;
(b) a two-fold real root, $b_{12}^{*} b_{21}^{*}=0$ and $\left(b_{12}^{*}\right)^{2}+\left(b_{21}^{*}\right)^{2}>0$;
(c) a two-fold real root and $b_{12}^{*} b_{21}^{*} \neq 0$,

(2) 2-dimensional if (2.2) has
(a) two real distinct roots and $b_{12}^{*}=b_{21}^{*}=0$;
(b) a pair of complex conjugate roots;
(c) a two-fold real root and $b_{12}^{*}=b_{21}^{*}=0$. 
Proof. We will carefully trace all theorems considered (Theorems 2.1-2.4) together with the case of a pair of complex conjugate roots uncovered by a theorem and our conclusion will hold on $\mathbb{Z}_{m+2}^{\infty}$ (some of the statements hold on a greater interval).

(a) Analysing the statement of Theorem 2.1 (the case (2.3) of two real distinct roots) we obtain the following subcases.

(a1) If $b_{11}^{*}=b_{22}^{*}=b_{21}^{*}=0, b_{12}^{*} \neq 0$, then the dimension of the space of solutions on $\mathbb{Z}_{m+2}^{\infty}$ equals $m+2$ since the last line in (2.13) uses only $m+2$ arbitrary parameters

$$
\varphi_{1}^{*}(0), \varphi_{2}^{*}(-m), \varphi_{2}^{*}(-m+1), \ldots, \varphi_{2}^{*}(0)
$$

(a2) If $b_{11}^{*}=b_{22}^{*}=b_{12}^{*}=0, b_{21}^{*} \neq 0$, then the dimension of the space of solutions on $\mathbb{Z}_{m+2}^{\infty}$ equals $m+2$ since the last line in (2.14) uses only $m+2$ arbitrary parameters

$$
\varphi_{1}^{*}(-m), \varphi_{1}^{*}(-m+1), \ldots, \varphi_{1}^{*}(0), \varphi_{2}^{*}(0) .
$$

(a3) If $b_{11}^{*}=b_{22}^{*}=b_{12}^{*}=b_{21}^{*}=0$, then the dimension of the space of solutions on $\mathbb{Z}_{m+2}^{\infty}$ equals 2 since the last line in (2.13) and in (2.14) uses only 2 arbitrary parameters

$$
\varphi_{1}^{*}(0), \quad \varphi_{2}^{*}(0)
$$

This means that all the cases considered are covered by conclusions (1a) and (2a) of Theorem 2.5.

(b) In the case (2.4) of two complex conjugate roots, we have $b_{11}^{*}=b_{22}^{*}=b_{12}^{*}=b_{21}^{*}=0$ and the formula (2.29) uses only 2 arbitrary parameters

$$
\varphi_{1}^{*}(0), \quad \varphi_{2}^{*}(0),
$$

for every $k \in \mathbb{Z}_{1}^{\infty}$. This is covered by case (2b) of Theorem 2.5 .

(c) Analysing the statement of Theorems 2.2 and 2.3 (the case (2.5) of two-fold real root), we obtain the following subcases.

(c1) If $b_{21}^{*}=0, b_{12}^{*} \neq 0$, then the dimension of the space of solutions on $\mathbb{Z}_{m+2}^{\infty}$ equals $m+2$ since the last line in (2.31) uses only $m+2$ arbitrary parameters

$$
\varphi_{1}^{*}(0), \varphi_{2}^{*}(-m), \varphi_{2}^{*}(-m+1), \ldots, \varphi_{2}^{*}(0)
$$

(c2) If $b_{12}^{*}=0, b_{21}^{*} \neq 0$, then the dimension of the space of solutions on $\mathbb{Z}_{m+2}^{\infty}$ equals $m+2$ since the last line in (2.32) uses only $m+2$ arbitrary parameters

$$
\varphi_{1}^{*}(-m), \varphi_{1}^{*}(-m+1), \ldots, \varphi_{1}^{*}(0), \varphi_{2}^{*}(0)
$$

(c3) If $b_{12}^{*}=b_{21}^{*}=0$, then the dimension of the space of solutions on $\mathbb{Z}_{m+2}^{\infty}$ equals 2 since the last line in (2.31) and in (2.32) uses only 2 arbitrary parameters

$$
\varphi_{1}^{*}(0), \quad \varphi_{2}^{*}(0)
$$


(c4) If $b_{12}^{*} b_{21}^{*} \neq 0$, then the dimension of the space of solutions on $\mathbb{Z}_{m+2}^{\infty}$ equals $m+2$ since the last line in (2.36) uses only $m+2$ arbitrary parameters

$$
C(-m), C(-m+1), \ldots, C(0), \varphi_{1}^{*}(0)
$$

where

$$
C(k):=\left[\varphi_{1}^{*}(k)+\frac{b_{12}^{*}}{b_{11}^{*}} \varphi_{2}^{*}(k)\right], \quad k \in \mathbb{Z}_{-m}^{0}
$$

The parameter $\varphi_{2}^{*}(0)$ cannot be seen as independent since it depends on the independent parameters $\varphi_{1}^{*}(0)$ and $C(0)$.

All the cases considered are covered by conclusions (1b), (1c), and (2c) of Theorem 2.5.

(d) Analysing the statement of Theorem 2.4 (The case (2.6) of two-fold real root), we obtain the following subcases.

(d1) If $b_{11}^{*}=b_{22}^{*}=b_{21}^{*}=0, b_{12}^{*} \neq 0$, then the dimension of the space of solutions on $\mathbb{Z}_{m+2}^{\infty}$ equals $m+2$ since the last line in (2.49) uses only $m+2$ arbitrary parameters

$$
\varphi_{1}^{*}(0), \varphi_{2}^{*}(-m), \varphi_{2}^{*}(-m+1), \ldots, \varphi_{2}^{*}(0),
$$

and the last line in (2.47) provides no new information.

(d2) If $b_{11}^{*}=b_{22}^{*}=b_{21}^{*}=b_{12}^{*}=0$, then the dimension of the space of solutions on $\mathbb{Z}_{m+2}^{\infty}$ equals 2 since, as follows from (2.49) and (2.47), there are only 2 arbitrary parameters

$$
\varphi_{1}^{*}(0), \quad \varphi_{2}^{*}(0)
$$

Both cases are covered by conclusions (1b) and (2c) of Theorem 2.5.

Since there are no cases other than the above cases $(a)-(d)$, the proof is finished.

Theorem 2.5 can be formulated simply as follows.

Theorem 2.6 (Main result). Let (1.1) be a system with weak delay and let (2.2) have both roots different from zero. Then the space of solutions, being initially $2(m+1)$-dimensional, is on $\mathbb{Z}_{m+2}^{\infty}$ only

(1) $(m+2)$-dimensional if $\left(b_{12}^{*}\right)^{2}+\left(b_{21}^{*}\right)^{2}>0$.

(2) 2-dimensional if $b_{12}^{*}=b_{21}^{*}=0$.

We omit the proofs of the following two theorems since again, they can be done in much the same way as Theorems 2.1-2.4.

Theorem 2.7. Let (1.1) be a system with weak delay and let (2.2) have a simple root $\lambda=0$. Then the space of solutions, being initially $2(m+1)$-dimensional, is either $(m+1)$-dimensional or 1 -dimensional on $\mathbb{Z}_{m+2}^{\infty}$. 
Theorem 2.8. Let (1.1) be a system with weak delay and let (2.2) have a two-fold root $\lambda=0$. Then the space of solutions, being initially $2(m+1)$-dimensional, turns into 0-dimensional space on $\mathbb{Z}_{1}^{\infty}$, namely, into the zero solution.

\section{Concluding Remarks}

To our best knowledge, weak delay was first defined in [4] for systems of linear delayed differential systems with constant coefficients. Nevertheless, separate particular examples can be found in various books concerning delayed differential equations. Let us summarize the advantage of investigating "weak" delayed systems in the plane. Such systems can be simplified and their solutions can be found in a simple explicit analytical form. In the case of ordinary differential systems with delay, to obtain the corresponding eigenvalues, it is sufficient to solve only a polynomial equation rather than a quasipolynomial one. In the case of discrete systems of two equations investigated in this paper in the "weak" case, to obtain the corresponding eigenvalues, it is sufficient to solve only polynomial equation of the second order rather than a polynomial equation of $2(m+1)$ th order. Note that results obtained can be directly used to investigate such asymptotic problems as boundedness or convergence of solutions (using different methods, such problems have recently been investigated, e.g., in $[5-11])$.

\section{Acknowledgments}

The first author was supported by the Grant 201/07/0145 of Czech Grant Agency (Prague), by the Council of Czech Government MSM 0021630503 and MSM 00216 30519. The third author was supported by the Council of Czech Government MSM 0021630503 and MSM 0021630529.

\section{References}

[1] J. Baštinec and J. Diblík, "Subdominant positive solutions of the discrete equation $\Delta u(k+n)=$ - p(k)u(k)," Abstract and Applied Analysis, vol. 2004, no. 6, pp. 461-470, 2004.

[2] J. Diblík and D. Ya. Khusainov, "Representation of solutions of linear discrete systems with constant coefficients and pure delay," Advances in Difference Equations, vol. 2006, Article ID 80825, 13 pages, 2006.

[3] S. N. Elaydi, An Introduction to Difference Equations, Undergraduate Texts in Mathematics, Springer, New York, NY, USA, 3rd edition, 2005.

[4] D. Ya. Khusainov, D. B. Benditkis, and J. Diblík, "Weak delay in systems with an aftereffect," Functional Differential Equations, vol. 9, no. 3-4, pp. 385-404, 2002.

[5] A. Boichuk and M. Růžičková, "Solutions of nonlinear difference equations bounded on the whole line," Folia Facultatis Scientiarium Naturalium Universitatis Masarykiana Brunensis. Mathematica, vol. 13, pp. 45-60, 2002.

[6] J. Čermák, “On matrix differential equations with several unbounded delays," European Journal of Applied Mathematics, vol. 17, no. 4, pp. 417-433, 2006.

[7] J. Čermák and M. Urbánek, "On the asymptotics of solutions of delay dynamic equations on time scales," Mathematical and Computer Modelling, vol. 46, no. 3-4, pp. 445-458, 2007.

[8] J. Diblík, "Anti-Lyapunov method for systems of discrete equations," Nonlinear Analysis: Theory, Methods E Applications, vol. 57, no. 7-8, pp. 1043-1057, 2004.

[9] M. Kipnis and D. Komissarova, "Stability of a delay difference system," Advances in Difference Equations, vol. 2006, Article ID 31409, 9 pages, 2006. 
[10] E. Liz and M. Pituk, "Asymptotic estimates and exponential stability for higher-order monotone difference equations," Advances in Difference Equations, vol. 2005, no. 1, pp. 41-55, 2005.

[11] Ch. G. Philos and I. K. Purnaras, "An asymptotic result for some delay difference equations with continuous variable," Advances in Difference Equations, vol. 2004, no. 1, pp. 1-10, 2004. 